\title{
Standardwerk in vierter Auflage
}

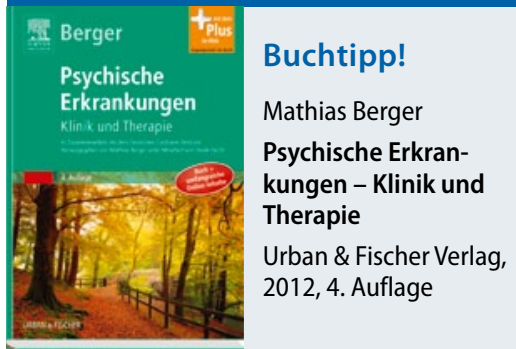

ISBN 978-3-437-22483-6; $149 €$ sich „Patient" und Psychotherapeut bei den Aufnahmen spürbar durch die Kamera beobachtet fühlten und manches mehr mit Blick auf den Zuschauer gesagt wird, kommt viel von dem herüber, was das Besondere an der Bergerschen Therapieschule ist. Auch für anders orientierte Psychotherapeuten sind diese Videos für die eigene Arbeit oder für eine kritische Auseinandersetzung sicher ein Gewinn. Dem Verlag ist allerdings zu raten, den Internetauftritt des Buches so zu verein- fachen, dass man mit zwei oder drei Klicks genau das bekommt, was man gesucht hat. Insgesamt ein wichtiges Buch für jeden Psychiater, vom Beginn seiner Weiterbildung bis zu den letzten Berufsjahren. Denn als fortlaufendes Lehrbuch kann es immer wieder neue Antworten auf neu gestellte Fragen geben. Ein Psychiater oder Psychotherapeut, der sich mit dem Buch vertraut gemacht hat, wird es stets griffbereit auf dem Schreibtisch haben wollen.

Uwe Henrik Peters, Köln
- Mit der 4. Auflage ist dieses Buch endgültig zu einem umfassenden Lehrbuch herangewachsen. Nicht nur die großen Psychosen, sondern jede Art von gestörter seelischer Gesundheit und deren Behandlung findet man in diesem Werk. In der Tat hat sich das Zuständigkeitsgebiet der Psychiater in den letzten Jahrzehnten ständig erweitert. Deshalb ist jedes einzelne der zahlreichen Kapitel knapp formuliert - anders wäre der Stoff nicht zu bewältigen. Der Sache nach vertritt das Buch eine Psychiatrie, die sich ab 1972 in den USA entwickelte, sich dann rasch über die westliche Welt verbreitete und sich als evidenzbasiert bezeichnet. Wohltuend wäre ein zusätzliches kleines philosophisches Kapitel, welches die Annahmen benennt, auf denen diese Psychiatrie aufbaut.

Es ist dem Herausgeber Mathias Berger gelungen, die Beiträge der mehr als 60 Mitarbeiter zu einem einheitlichen Werk zu vereinigen. So weit sich trotzdem noch Fehler oder Inkompatibilitäten eingeschlichen haben sollten, werden die Benutzer aufgefordert, sich unmittelbar mit den Verfassern in Verbindung zu setzen.

Da ein solches Buch rasch veraltet, sind gegenüber der 3. Auflage (2009) einige Neuerungen eingeführt worden: Wer das Buch besitzt, bekommt Zugang zu Ergänzungen, Erweiterungen und Korrekturen im Internet. Dort findet man zum Beispiel auch sorgfältig formulierte Zusatzkapitel zu häufig diskutierten Problembereichen, deren Platz im Gesamtsystem noch unsicher ist. Als Beispiele seien hier das „Burnout-Syndrom“, die „posttraumatische Verbitterungsstörung nach Linden“, und das „Stalking“ genannt.

Man kann auch Videos zu Therapiesitzungen abrufen. Auf den Videos stellen Schauspieler als Patienten die Szenen nach. Und obwohl

\section{Fit für die neurologische Facharztprüfung}

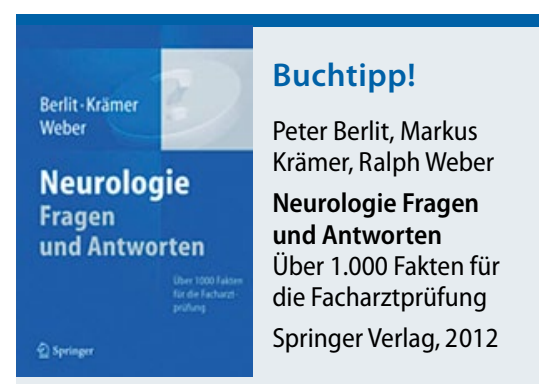

ISBN 978-3-642-29731-1; 32,95€

— Richtig oder falsch? „Bei der Akuttherapie des ischämischen Schlaganfalls sollte der Blutdruck erst ab Werten über 220/120
mmHg gesenkt werden.“ „Eine Migräneaura kann auch isoliert ohne Kopfschmerzen auftreten“.,Die okuläre Myasthenie, bei der nur die Augenmuskeln betroffen sind, macht $50 \%$ aller Myasthenien aus."

Wer die Antwort auf diese Fragen aus dem Ärmel schütteln kann, ist gut auf die Facharztprüfung Neurologie vorbereitet. Allen anderen sei das Buch,NNeurologie Fragen und Antworten" empfohlen. Hier kann man sein Wissen anhand von Multiple-Choice-Fragen systematisch testen. Im Anhang finden sich außerdem Auszüge wichtiger klinischer Studien mit den dazugehörigen Kernaussagen. Mit prägnanten Kommentaren zu allen Antworten kann sich so jeder Assistenzarzt gut auf die FacharztPrüfung vorbereiten. red

\section{Der "Doose" in neuer Auflage}

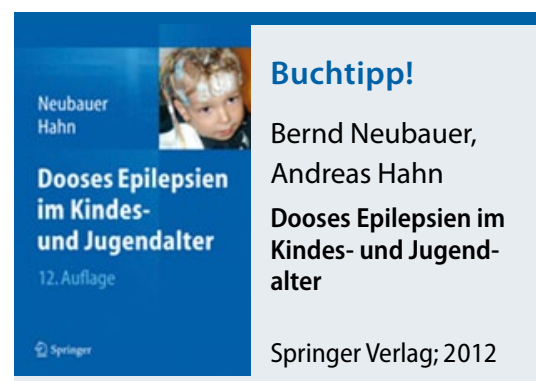

ISBN 978-3-642-29437-2; 59,95€

Bestellmöglichkeit: www.springer.com

— Seit über 40 Jahren eine feste Größe, wenn es um zerebrale Anfälle und Epilepsien bei
Kindern und Jugendlichen geht - "der Doose". Dieses Buch bietet alles, was Pädiater und Neuropädiater zu diesem Thema wissen sollten, verständlich und praxisnah. Es beinhaltet zum Beispiel die neue Klassifikation der Anfallssymptomatologie, stellt Epilepsien mit klinischen Symptomen, speziellen elektroenzephalografischen Merkmalen und besonderen Verlaufscharakteristika dar sowie MRT und EEG-Aufnahmen gegenüber. Zudem sind die Kernaussagen zu den Syndromen übersichtlich zusammengefasst. Neu in der 12. Auflage sind Informationen zu Genetik, Epilepsien bei Stoffwechselerkrankungen, Hirnfehlbildungen, Epilepsiechirurgie und alternativen Behandlungsformen plus eine Übersicht über Antiepileptika. red 